Artículo original

\title{
Eficacia de una estrategia combinada para mejorar el control del colesterol unido a lipoproteínas de baja densidad en pacientes con hipercolesterolemia. Ensayo clínico aleatorizado
}

\author{
Ignacio Párraga-Martínez ${ }^{\mathrm{a}, *}$, Francisco Escobar-Rabadán ${ }^{\mathrm{b}}$, Joseba Rabanales-Sotos ${ }^{\mathrm{c}}$, \\ Fernando Lago-Deibe ${ }^{\mathrm{d}}$, Juan M. Téllez-Lapeira ${ }^{\mathrm{e}}$, Alejandro Villena-Ferrer ${ }^{\mathrm{f}}$, Mariano Blasco-Valle ${ }^{\mathrm{g}}$,

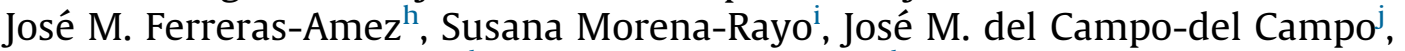 \\ M. Candelaria Ayuso-Raya ${ }^{\mathrm{b}}$ y José J. Pérez-Pascual ${ }^{\mathrm{b}}$
}

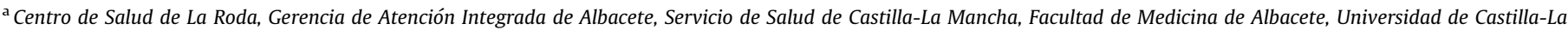 \\ Mancha, Albacete, España \\ ${ }^{\mathrm{b}}$ Centro de Salud Zona IV, Servicio de Salud de Castilla-La Mancha, Albacete, España \\ ${ }^{\mathrm{c}}$ Facultad de Enfermería de Cuenca, Universidad de Castilla-La Mancha, Cuenca, España \\ ${ }^{\mathrm{d}}$ Centro de Salud de Sárdoma, Servicio de Salud de Galicia, Vigo, Pontevedra, España \\ e Centro de Salud Zona V-B, Servicio de Salud de Castilla-La Mancha, Albacete, España \\ ${ }^{\mathrm{f}}$ Centro de Salud de San Clemente, Servicio de Salud de Castilla-La Mancha, San Clemente, Cuenca, España \\ ${ }^{\mathrm{g}}$ Centro de Salud Delicias Sur, Servicio de Salud Aragón, Zaragoza, España \\ ${ }^{\mathrm{h}}$ Servicio de Urgencias, Hospital Royo Villanova, Servicio Aragonés de Salud, Zaragoza, España

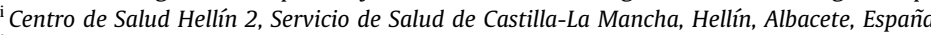 \\ ${ }^{\mathrm{j}}$ Centro de Salud de Almansa, Servicio de Salud de Castilla-La Mancha, Almansa, Albacete, España
}

Historia del artículo:

Recibido el 18 de septiembre de 2016

Aceptado el 15 de marzo de 2017

\section{Palabras clave:}

Hipercolesterolemia

Cumplimiento terapéutico

Atención primaria de salud

Ensayo clínico

\section{RES U M E N}

Introducción y objetivos: Intervenciones diferentes pueden mejorar el control del colesterol unido a lipoproteínas de baja densidad (cLDL). El objetivo principal era evaluar la eficacia de una intervención combinada para mejorar el control del cLDL de pacientes con hipercolesterolemia. También se evaluó su eficacia para mejorar el cumplimiento (farmacológico, dieta y ejercicio).

Métodos: Ensayo clínico aleatorizado, de grupos paralelos y multicéntrico (atención primaria) que incluyó a 358 adultos diagnosticados de hipercolesterolemia con tratamiento previo farmacológico o no. Se comparó a 178 sujetos que recibieron intervención combinada (material escrito, tarjetas autocumplimentadas y mensajes al móvil) frente a 178 controles. La variable principal de resultado fue la proporción de sujetos con adecuado control del cLDL (valores recomendados en las guías europeas de dislipemias y riesgo cardiovascular) a los 24 meses.

Resultados: El grupo de intervención mostró una reducción media del cLDL significativamente superior a los 24 meses respecto al control, 23,8 mg/dl (IC95\%, 17,5-30,1) y 14,6 mg/dl (IC95\%, 8,9-20,4), respectivamente $(p=0,034)$. El promedio de la reducción del cLDL fue del 13,1 $\pm 28,6 \%$. La proporción de sujetos con adecuado control al año fue significativamente superior en el grupo de intervención (43,7 frente a $30,1 \% ; p=0,011 ; R R=1,46)$. En el grupo de intervención, el cumplimiento farmacológico fue significativamente superior (77,2 frente a $64,1 \% ; p=0,029)$ y de la práctica de ejercicio $(64,9$ frente a $35,8 \% ; p<0,001)$, aunque no de la dieta.

Conclusiones: La intervención combinada consigue una reducción significativa de las cifras de cLDL (superior al 13\% al cabo de 2 años) y mejora el grado de control de pacientes con hipercolesterolemia al año.

(c) 2017 Sociedad Española de Cardiología. Publicado por Elsevier España, S.L.U. Todos los derechos reservados.

Efficacy of a Combined Strategy to Improve Low-density Lipoprotein Cholesterol Control Among Patients With Hypercholesterolemia: A Randomized Clinical Trial

A B S T R A C T

Introduction and objectives: Several interventions can improve low-density lipoprotein cholesterol (LDL-C) control. Our main objective was to evaluate the efficacy of a combined intervention to improve LDL-C control in patients with hypercholesterolemia. The study also assessed the efficacy of the intervention in improving adherence (pharmacological, diet, and exercise).
Hypercholesterolemia

Medication adherence

Primary health care

Clinical trial

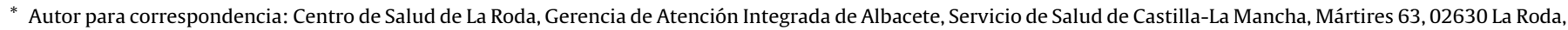
Albacete, España.

Correo electrónico: iparraga@sescam.jccm.es (I. Párraga-Martínez).
} 
Methods: A multicenter, parallel group, randomized clinical trial (primary care) was conducted in 358 adults diagnosed with hypercholesterolemia, whether receiving prior drug therapy or not. We compared 178 participants who received the combined intervention (written material, self-completed registration cards, and messages to mobile telephones) with 178 controls. The main outcome variable was the proportion of participants with adequate LDL-C control (target levels of the European guidelines on dyslipidemia and cardiovascular risk) at 24 months.

Results: At 24 months, the mean reduction in LDL-C was significantly higher in the intervention group (23.8 $\mathrm{mg} / \mathrm{dL}$ [95\%CI, 17.5-30.1]) than in the control group $(14.6 \mathrm{mg} / \mathrm{dL}$ [95\%Cl, 8.9-20.4]; $P=.034)$. The mean LDL-C decrease was $13.1 \% \pm 28.6 \%$. At 1 year, the proportion of participants with adequate control was significantly higher in the intervention group than in the control group $(43.7 \%$ vs $30.1 \% ; P=.011$; RR 1.46). Adherence was significantly higher in the intervention group, both to drug therapy $(77.2 \%$ vs $64.1 \%$; $P=.029)$ and exercise $(64.9 \%$ vs $35.8 ; P<.001)$, but not to diet.

Conclusions: The combined intervention significantly reduced LDL-C (by more than $13 \%$ at 2 years) and improved the degree of LDL-C control in patients with hypercholesterolemia at 1 year.

Full English text available from: www.revespcardiol.org/en

(c) 2017 Sociedad Española de Cardiología. Published by Elsevier España, S.L.U. All rights reserved.

\author{
Abreviaturas \\ cLDL: colesterol unido a lipoproteínas de baja densidad \\ $\mathrm{CT}$ : colesterol total \\ $\mathrm{RCV}$ : riesgo cardiovascular
}

\section{INTRODUCCIÓN}

La prevalencia de hipercolesterolemia en España se sitúa entre el 20 y el 50\%, en función de las concentraciones plasmáticas de colesterol consideradas ${ }^{1,2}$.

Hay consenso acerca de los beneficios de la dieta, la práctica de ejercicio y el uso de fármacos en prevención primaria, y sobre todo en prevención secundaria, para un mejor control de los pacientes con hipercolesterolemia ${ }^{3-5}$. A pesar de las recomendaciones y de los nuevos fármacos hipolipemiantes, las cifras de colesterol total (CT) y colesterol unido a lipoproteínas de baja densidad (cLDL) superan los objetivos recomendados tanto en España como en Europa $^{2,6-9}$.

Uno de los factores limitantes para alcanzar los objetivos en pacientes hipercolesterolémicos es el incumplimiento terapéutico, que afecta tanto al uso de medicamentos como al resto de las recomendaciones sobre estilos de vida ${ }^{10}$.

Ante esta situación se han utilizado diferentes estrategias para mejorar el cumplimiento y, por tanto, el control de estos pacientes. En pacientes con factores de riesgo como la hipertensión se ha mejorado el cumplimiento mediante la combinación de entrega de material escrito, realización de llamadas telefónicas y envío de mensajes postales informativos sobre la enfermedad ${ }^{11,12}$. En sujetos con dislipemia se han demostrado mejoras del cumplimiento con recordatorios telefónicos ${ }^{13,14}$ y mediante tarjetas con información sobre el tratamiento ${ }^{15}$. Los estudios sobre cumplimiento en dislipemia indican que, para alcanzar objetivos de control adecuados, es necesario mejorar tanto el cumplimiento farmacológico como la adherencia al resto de recomendaciones ${ }^{10}$.

Al considerar que la combinación de estrategias para mejorar la adherencia muestra mejores resultados que las intervenciones individuales ${ }^{16,17}$, se planteó utilizar una estrategia que incluyera intervenciones con eficacia demostrada en mejorar el cumplimiento $^{13-15}$. Así, el objetivo principal de este estudio es evaluar la eficacia de una intervención para mejorar el grado de control de los pacientes hipercolesterolémicos; consistente en una estrategia combinada que incluye la entrega de material informativo escrito, mensajes de texto y tarjetas de registro del grado de cumplimiento, como medidas complementarias a la actuación habitual en la consulta. Además, se evaluó la eficacia para mejorar el cumplimiento (farmacológico, dieta y ejercicio).

\section{MÉTODOS}

Se realizó un ensayo clínico aleatorizado de grupos paralelos y multicéntrico. Estudio registrado en ClinicalTrials.gov (NCT02314663). Se seleccionó a los participantes en las consultas de 8 centros de salud de 3 áreas sanitarias de 3 comunidades autónomas españolas: Castilla-La Mancha (Albacete), Aragón (Zaragoza) y Galicia (Vigo). Los criterios de inclusión fueron: sujetos diagnosticados previamente de hipercolesterolemia definida $(\mathrm{CT} \geq 250 \mathrm{mg} / \mathrm{dl})^{18}$, con su tratamiento habitual (farmacológico o no), $\geq 18$ años y usuarios de los centros participantes. Se excluyó a aquellos pacientes con impedimento para realizar el seguimiento durante la intervención (analfabetos y no usuarios de teléfono móvil), incapacidad física para colaborar y a los que presentaban una enfermedad crónica grave orgánica o psiquiátrica que les impidiera acudir a las visitas de seguimiento. Todos los sujetos firmaron el consentimiento informado tras la explicación adecuada del estudio. El Comité Ético de Investigación Clínica del Área Sanitaria de Albacete aprobó el estudio y se ajustó a las directrices éticas para ensayos clínicos (Real Decreto 223/2004) y la Declaración de Helsinki.

Se calculó el tamaño muestral necesario para un análisis mediante contraste bilateral y se consideró una diferencia clínicamente relevante del $20 \%$ en la proporción de sujetos que alcanzan los objetivos de control lipídico: $55 \%$ en el grupo control ${ }^{19}$ y $75 \%$ en el grupo sometido a la intervención. Con un $90 \%$ de potencia estadística y un $5 \%$ de probabilidad de error alfa, se estimó un tamaño muestral de 155 sujetos por grupo (310 total). Tras considerar una proporción esperada de pérdidas del 15\%, el tamaño definitivo por grupo fue de 179 sujetos (total, 358). De los 379 sujetos evaluados para la selección, 21 no aceptaron participar (tasa aceptación: 94,5\%) (figura 1). El periodo de reclutamiento abarcó desde marzo a diciembre de 2013.

En cuanto a la selección de participantes, por razones estratégicas se eligieron 8 zonas básicas de salud de las áreas sanitarias de Albacete, Zaragoza y Vigo. Para evitar un sesgo de contaminación, los participantes del grupo control pertenecían a zonas diferentes a las del grupo experimental. Por tanto, la aleatorización de participantes se realizó de forma centralizada en función de las zonas de salud (aleatorización de Efrom) y la efectuó un investigador ajeno a las entrevistas y a los análisis. Los sujetos se seleccionaron de forma consecutiva. 


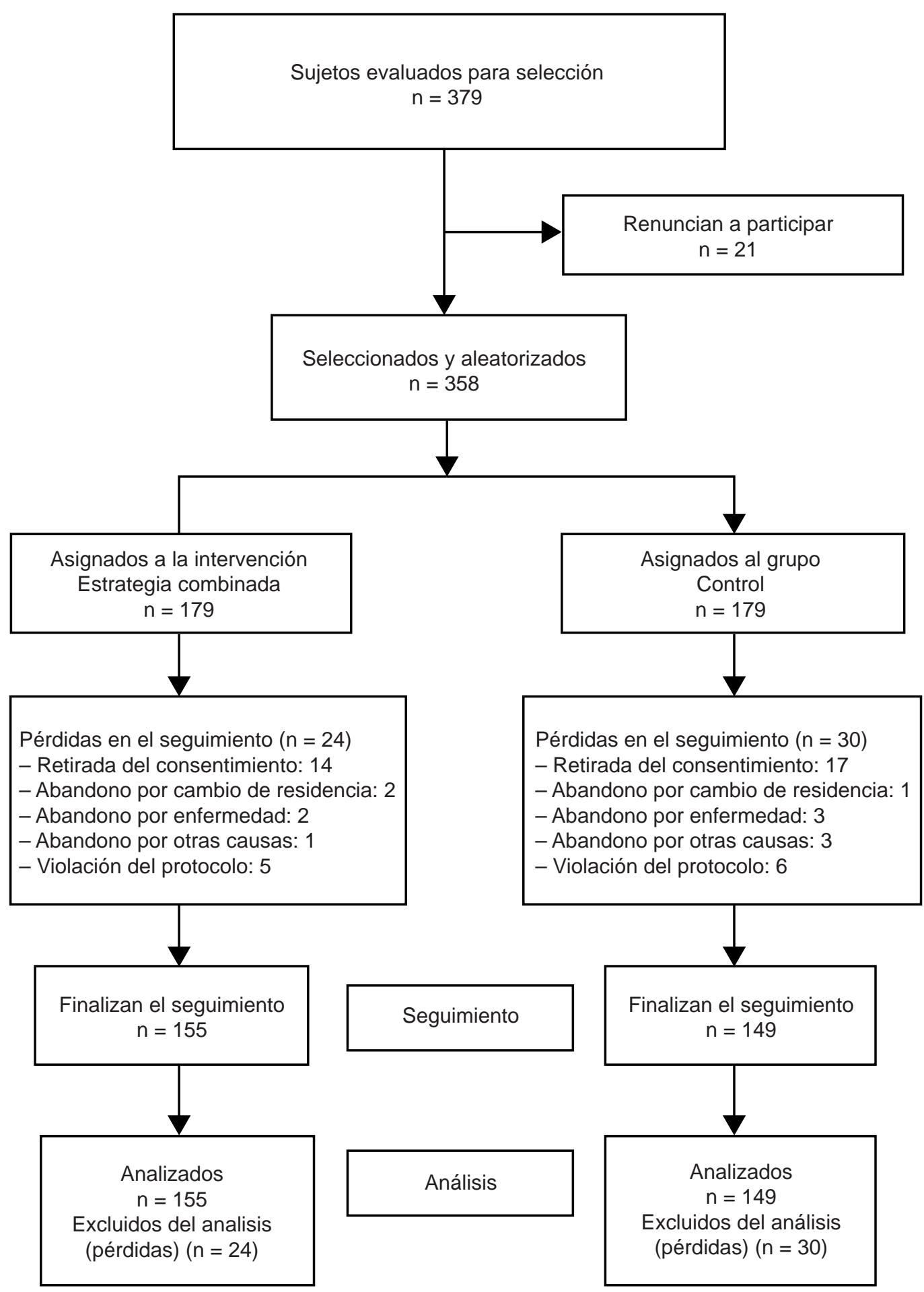

Figura 1. Esquema del estudio.

Respecto a la intervención, se facilitó a los participantes del grupo que la recibió: a) material escrito con información sobre la enfermedad y su tratamiento (facilitado en cada visita) (anexo 1 y anexo 2 del material suplementario); b) mensajes de texto al teléfono móvil con resúmenes de recomendaciones, recordatorios de fechas de próximas citas y convocatorias para nuevas citas en caso de no asistencia (durante periodos entre visitas) (anexo 3 del material suplementario), y c) tarjetas autocumplimentadas sobre el seguimiento de las recomendaciones (durante todo el seguimiento) (anexo 4 del material suplementario). Ambos grupos (intervención y control) recibieron las recomendaciones habituales según las guías de práctica clínica europeas sobre tratamiento de hipercolesterolemia y riesgo cardiovascular $(\mathrm{RCV})^{3,20}$.

El seguimiento de los sujetos se realizó durante un periodo de 2 años que finalizó en diciembre de 2015 . Una vez seleccionados, y tras otorgar su consentimiento, se citó a los pacientes para la visita inicial. En esta visita se completaron los datos de anamnesis, exploración física y analítica. Se les ofrecieron las recomendaciones habituales, farmacológicas y no farmacológicas, para su tratamiento. Junto con las tarjetas para el seguimiento, a los participantes del grupo de intervención se les entregó documentación escrita sobre la enfermedad y se les informó acerca de la 
periodicidad y el contenido de los mensajes de texto que recibieron en su teléfono móvil. Los mensajes recordatorios del tratamiento de su patología se remitieron cada 15 días y los de asistencia a citas pendientes o ausentes se enviaron en función de las fechas de seguimiento. Tras la visita inicial, en ambos grupos se realizaron 5 visitas de seguimiento al cabo de 2, 6, 12, 18 y 24 meses. Las variables se registraron en cada visita, aunque únicamente se obtuvieron datos sobre satisfacción durante la visita final del grupo de intervención.

La variable principal de resultado fue la proporción de sujetos que alcanzaron los valores de cLDL marcados como objetivo por las guías europeas de dislipemias ${ }^{3}$ y $\mathrm{RCV}^{20}$ a lo largo de un periodo de seguimiento de 24 meses. Las cifras plasmáticas indicadas como objetivos fueron: a) cLDL $<115 \mathrm{mg} / \mathrm{dl}$ para pacientes sin enfermedad cardiovascular establecida ni diabetes mellitus y con RCV bajo o moderado (SCORE $<5 \%$ ); b) $\mathrm{cLDL}<100 \mathrm{mg} / \mathrm{dl}$ para pacientes sin enfermedad cardiovascular establecida ni diabetes mellitus con daño orgánico y con RCV alto (SCORE $\geq 5 \% \mathrm{y}<10 \%$ ), y ce:italic $>$ c) cLDL $<70 \mathrm{mg} / \mathrm{dl}$ para pacientes con diabetes mellitus o enfermedad cardiovascular establecida y RCV muy alto (SCORE $\geq$ $10 \%)^{3,20}$. También se midió el perfil lipídico (CT, cLDL, colesterol unido a lipoproteínas de alta densidad y triglicéridos) y su variación en cada visita a todos los participantes y se les informó del resultado. Las determinaciones analíticas se tomaron de sangre venosa tras un ayuno $\geq 12 \mathrm{~h}$. El CT se determinó mediante el método CHODPAP y el cLDL con el de Friedewald. Otras variables determinadas fueron: características sociodemográficas (edad, sexo, estado civil, nivel de instrucción y categoría social ${ }^{21}$ ), consumo de fármacos hipolipemiantes (tipo, dosificación), cumplimiento del tratamiento hipolipemiante (test de MoriskyGreen $^{22}$ : considera buena adherencia la respuesta adecuada a las 4 preguntas dicotómicas del cuestionario referidas al olvido de una toma y a la adecuación a la pauta), acontecimientos adversos, adherencia a recomendaciones sobre estilos de vida (MoriskyGreen adaptado), actividad física, hábitos alimentarios, aparición de eventos cardiovasculares (cardiopatía isquémica, enfermedad cerebrovascular aterotrombótica y enfermedad arterial periférica), datos antropométricos, hábito tabáquico, presión arterial sistólica y diastólica, RCV (SCORE para países de bajo RCV y REGICOR) ${ }^{23}$, problemas de salud (segunda edición de la Clasificación Internacional de Atención Primaria, Organización Mundial de Médicos de Familia), consumo de otros medicamentos y grado de satisfacción con la estrategia combinada mediante un cuestionario de satisfacción (escala Likert con 5 opciones de respuesta que van desde $1=$ "muy insatisfecho» a 5 = «muy satisfecho»). Las causas de finalización del estudio fueron: periodo de observación completo (2 años), violación del protocolo, enfermedad intercurrente que imposibilitara continuar la intervención y abandono del paciente/ retirada del consentimiento. No se realizaron cambios en el protocolo durante el trascurso del ensayo.

Respecto al análisis estadístico, en ambos grupos se compararon las variables de interés y variables de estratificación y potencialmente confusoras al inicio del estudio. Se comprobó la homogeneidad de grupos para los valores basales de las variables ( $t$ de Student, $\chi^{2}$ ). Posteriormente, se clasificó a los sujetos de ambos grupos en diferentes niveles de reducción o control del cLDL y se realizó un primer análisis crudo para evaluar los siguientes parámetros junto a sus correspondientes intervalos de confianza del 95\% (IC95\%): aumento absoluto de beneficio, aumento relativo de beneficio y número necesario a tratar. Además, se describió y comparó la incidencia de la variable de resultado (proporción de sujetos con control adecuado del cLDL) en ambos grupos en cada periodo de seguimiento (comparación de proporciones: $\chi^{2}$ ). La comparación de variaciones a lo largo del estudio en el perfil lipídico de cada grupo se realizó mediante la prueba de la t de Student o su alternativa no paramétrica (U de Mann-Whitney). La modificación de parámetros en cada grupo se analizó mediante la prueba de la $t$ para medidas repetidas. La posible existencia de factores de confusión o la interacción de otras variables en la relación entre la intervención propuesta y las variables de resultado se evaluó mediante modelos de regresión logística (variable dependiente: control de los parámetros lipídicos). Para las variables continuas (reducción del cLDL), la evaluación se efectuó mediante regresión lineal múltiple. El análisis de eficacia se realizó por intención de tratar. En este análisis se incluyó a todos los sujetos evaluados a los 12 y 24 meses. Los análisis se llevaron a cabo mediante el programa SPSS (versión 20.0).

\section{RESULTADOS}

De los 358 sujetos que iniciaron el estudio, 155 y 149 completaron el seguimiento en el grupo de intervención y control, respectivamente. Los grupos no fueron diferentes respecto al porcentaje de sujetos que completaron el estudio (86,6 frente $83,2 \% ; \mathrm{p}=0,376$ ). La figura 1 muestra la distribución de los pacientes perdidos.

Las características basales de ambos grupos se muestran en la tabla 1. Los grupos eran homogéneos al inicio del estudio.

En la tabla 2 se muestran las diferencias intragrupales y entre los grupos de intervención y control respecto a los parámetros lipídicos a los 12 y 24 meses. Al comparar la modificación de las cifras de los parámetros lipídicos entre ambos grupos durante el seguimiento, se observó una mayor reducción media de cLDL y CT en el grupo de intervención tanto al año como a los 2 años. El cLDL medio (mg/dl) al año fue de $124,9 \pm 37,0$ y $119,5 \pm 36,5$ en el grupo control e intervención, respectivamente, y a los 24 meses de $122,7 \pm 35,3$ y $117,1 \pm 33,4$. Al comparar ambos grupos respecto a la modificación de parámetros antropométricos, presión arterial y RCV durante el seguimiento, no hubo diferencias estadísticamente significativas (tabla 2).

El grupo de intervención mostró una reducción media del cLDL respecto a su cifra inicial del 12,8 frente al 5,2\% del grupo control (IC95\%, 2,1-13,3\%; p = 0,007) a los 12 meses y del 13,1 frente al 6,4\% (IC95\%, 0,4-13,0\%; p = 0,038) a los 24 meses.

La proporción de sujetos que redujo el cLDL fue significativamente superior en el grupo de intervención a los 12 meses (riesgo relativo $[R R]=1,30$; IC95\%, 1,11-1,51) y a los 24 meses $(R R=1,17$; IC95\%, 1,02-1,36). La proporción de sujetos con cifras controladas de cLDL fue significativamente superior en el grupo de intervención respecto al grupo control a los 12 meses ( $R R=1,46$; IC95\%, 1,08-1,97). También fue mayor a los 24 meses, aunque sin alcanzar significación estadística (43,4\% frente a 34,7\%; p = 0,119). Al año, el aumento absoluto de beneficio por alcanzar el control del cLDL fue del 14\% (IC95\%, 3-24\%); el aumento relativo de beneficio del 32\% (IC95\%, 8-49\%), y el número necesario a tratar de 7 (IC95\%, 4-32). Al realizar un análisis post-hoc, considerándose únicamente el subgrupo de participantes con RCV bajo o moderado (SCORE $<5 \%$ ), $5 \%)$, la proporción de sujetos con cLDL controlado fue significativamente superior en el grupo de intervención tanto a los 12 ( $R R=1,41$; IC95\%, 1,02-1,94) como a los 24 meses ( $R R=1,39$; IC95\%, 1,02-1,90). Esto no se observó en sujetos con RCV alto o muy alto. La figura 2 (anexo 5 del material suplementario, pacientes con objetivo logrado según su grupo de RCV) muestra el porcentaje de pacientes del grupo de intervención que alcanzó el objetivo terapéutico en cada subgrupo de RCV al inicio y a los 12 y 24 meses.

Respecto al cumplimiento del tratamiento con estatinas, fue significativamente superior en el grupo de intervención tanto al año ( $R R=1,21$; IC95\%, 1,02-1,43) como a los 2 años $(R R=1,20$; IC95\%, 1,02-1,427). También hubo mayor adherencia a las recomendaciones sobre ejercicio en este grupo al año $(R R=1,58$; IC95\%, 1,24-2,01) y a los 24 meses (RR = 1,81; IC95\%, 1,42-2,32). No 
Tabla 1

Características basales de los participantes que finalizaron el estudio y homogeneidad de ambos grupos

\begin{tabular}{|c|c|c|c|c|}
\hline Características & $\begin{array}{l}\text { Muestra total } \\
(\mathrm{n}=304)\end{array}$ & $\begin{array}{l}\text { Grupo de intervención } \\
(n=155)\end{array}$ & $\begin{array}{l}\text { Grupo control } \\
(\mathrm{n}=149)\end{array}$ & $\mathrm{p}$ \\
\hline Edad (años) media (DE) & $59,1 \pm 9,4$ & $58,9 \pm 10,4$ & $59,3 \pm 8,4$ & 0,738 \\
\hline \multicolumn{5}{|l|}{ Edad (años) } \\
\hline$<50$ & 16,1 & 20,0 & 12,1 & \multirow[t]{3}{*}{0,172} \\
\hline $50-60$ & 35,9 & 34,2 & 37,7 & \\
\hline$>60$ & 48,0 & 45,8 & 50,3 & \\
\hline Mujeres \% & 54,9 & 56,1 & 53,7 & 0,669 \\
\hline Casados o unión estable & 79,9 & 80,0 & 79,9 & 0,977 \\
\hline Estudios secundarios o superiores & 31,2 & 28,4 & 34,2 & 0,272 \\
\hline Clases sociales $v-V I I^{a}$ & 68,2 & 65,6 & 70,9 & 0,317 \\
\hline Enfermedad crónica en curso ${ }^{\mathrm{b}}$ & 73,6 & 71,6 & 75,7 & 0,423 \\
\hline Consumo de algún fármaco no hipolipemiante & 77,6 & 74,2 & 81,2 & 0,142 \\
\hline Consumo de estatinas & 68,1 & 64,5 & 71,8 & 0,172 \\
\hline Actividad física ${ }^{\mathrm{c}}$ & 57,6 & 60,6 & 54,4 & 0,269 \\
\hline Tiempo sedentario/semana $(\mathrm{min})^{\mathrm{d}}$ & $233,1 \pm 129,6$ & $232,9 \pm 136,9$ & $233,3 \pm 122,1$ & 0,977 \\
\hline Síndrome metabólico (NCEP-ATP III) & 29,0 & 25,0 & 33,1 & 0,124 \\
\hline Fumadores & 22,7 & 23,9 & 21,5 & 0,618 \\
\hline Diabéticos & 17,1 & 14,8 & 19,5 & 0,284 \\
\hline Hipertensos & 51,6 & 49,7 & 53,7 & 0,484 \\
\hline Cardiopatía isquémica & 4,9 & 4,5 & 4,7 & 0,940 \\
\hline Prevención primaria & 93,1 & 91,0 & 95,3 & 0,136 \\
\hline Obesos o con sobrepeso (IMC $\left.\geq 25 \mathrm{~kg} / \mathrm{m}^{2}\right)$ & 84,5 & 85,2 & 83,9 & 0,760 \\
\hline Control del valor plasmático de cLDL & 28,1 & 26,5 & 29,7 & 0,525 \\
\hline CT en plasma medio $(\mathrm{mg} / \mathrm{dl})$ & $224,7 \pm 42,7$ & $227,4 \pm 42,4$ & $221,9 \pm 42,8$ & 0,269 \\
\hline cLDL en plasma medio $(\mathrm{mg} / \mathrm{dl})$ & $139,1 \pm 38,2$ & $141,0 \pm 36,7$ & $137,3 \pm 39,0$ & 0,354 \\
\hline cHDL en plasma medio $(\mathrm{mg} / \mathrm{dl})$ & $57,3 \pm 14,7$ & $58,9 \pm 14,8$ & $55,7 \pm 14,5$ & 0,071 \\
\hline Triglicéridos en plasma medio (mg/dl) & $140,5 \pm 73,3$ & $135,2 \pm 65,1$ & $146,1 \pm 80,8$ & 0,196 \\
\hline
\end{tabular}

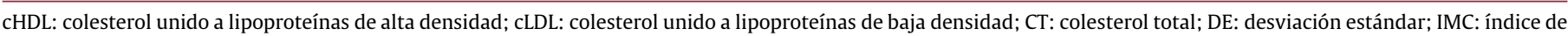
masa corporal; NCEP-ATP III: National Cholesterol Education Program-Adult Treatment Panel III.

Salvo otra indicación, los valores expresan porcentaje o media \pm desviación estándar.

a Trabajadores manuales semicualificados o no cualificados (de la industria, comercio, servicios y sector primario) y amas de casa.

b Enfermedad crónica en curso: presencia de enfermedad crónica incluida en la segunda edición de la Clasificación Internacional de Atención Primaria y diagnosticada antes del inicio del estudio y que continua existiendo en ese momento.

c Actividad física varias veces por semana o a diario (se pregunta si realiza ejercicio regularmente con las opciones de respuesta: nunca, 1 vez al mes, 1 vez por semana, varias veces por semana, a diario).

d Minutos sentado a la semana (Cuestionario Internacional de Actividad Física, versión corta).

hubo diferencias intergrupales estadísticamente significativas respecto al cumplimiento de las recomendaciones dietéticas, aunque fue superior en el grupo de intervención. En la tabla 3 se muestra la evolución de los porcentajes de cumplimiento durante el seguimiento.

En la tabla 4 se presenta la distribución de pacientes en función de fármaco consumido al inicio del estudio y los cambios de tratamiento durante el seguimiento, sin diferencias entre ambos grupos. No se observaron diferencias respecto a los efectos adversos de las estatinas ( 7 en el grupo de intervención, 10 en el grupo control). Ningún paciente manifestó efectos adversos relacionados con la intervención.

Respecto a la satisfacción con la intervención, el 74,0\% (IC95\%, $66,8-81,3 \%$ ) y el $90,8 \%$ (IC95\%, 85,9-95,7\%) de los que la recibieron manifestó estar satisfecho o muy satisfecho con ella al año y a los 2 años, respectivamente.

En la tabla del material suplementario se presentan los factores relacionados en el análisis multivariante con una mayor reducción de las cifras de cLDL (regresión lineal múltiple) a los 12 y 24 meses ("variables y reducción de cLDL»). En la tabla 5 se muestran las variables relacionadas en la regresión logística con un adecuado control de los valores de cLDL a los 12 meses.

\section{DISCUSIÓN}

El objetivo primordial del tratamiento de la dislipemia es la reducción de los valores de $\mathrm{CLDL}^{3-5}$ y alcanzar los objetivos terapéuticos recomendados por la guías de práctica clínica ${ }^{4,5}$, aunque existe un amplio margen de mejora respecto a su control $^{6,24}$. Los resultados del presente estudio muestran que, en comparación con las actuaciones recomendadas por la guías de práctica clínica en la consulta, la intervención combinada (entrega de material escrito, mensajes de texto y tarjetas de registro del cumplimiento) añadida a esas actuaciones se asoció a una mayor reducción del cLDL y a un mejor cumplimiento del tratamiento hipolipemiante y de las recomendaciones sobre ejercicio a los 2 años; mientras que únicamente se relacionó con superior control del CLDL al año de seguimiento, pues esa superioridad no resultó significativa a los 24 meses. Estos resultados podrían deberse tanto a la dificultad para lograr los objetivos en pacientes con RCV más elevado -indicada en estudios nacionales y europeos ${ }^{25,26}$ - como a las cifras consideradas objetivo para los grupos de riesgo más altos; puesto que, si se analiza solo el subgrupo de pacientes con RCV moderado o bajo (SCORE $<5 \%$ a 10 años), se ha observado superior control en el grupo de intervención a los 24 meses. Además, podría 
Tabla 2

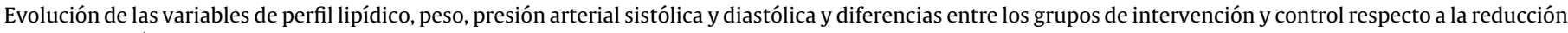
de dichos parámetros al cabo de 12 y 24 meses

\begin{tabular}{|c|c|c|c|c|c|c|}
\hline \multirow[t]{3}{*}{ Variables } & \multicolumn{3}{|c|}{12 meses } & \multicolumn{3}{|c|}{24 meses } \\
\hline & \multirow{2}{*}{$\frac{\text { Modificación desde basal }}{\text { Media (IC95\%) }}$} & \multicolumn{2}{|c|}{ Diferencia de reducción } & \multirow{2}{*}{$\frac{\text { Modificación desde basal }}{\text { Media (IC95\%) }}$} & \multicolumn{2}{|c|}{ Diferencia de reducción } \\
\hline & & Media (IC95\%) & $\mathrm{p}$ & & Media (IC95\%) & $\mathrm{p}$ \\
\hline \multicolumn{7}{|l|}{$c L D L(m g / d l)$} \\
\hline Intervención & $-21,4(-26,8 \mathrm{a}-16,0)$ & $9,3(1,5$ a 17,1$)$ & \multirow{2}{*}{$0,019^{*}$} & $-23,8(-30,1 \mathrm{a}-17,5)$ & $9,2(0,7$ a 17,7$)$ & \multirow{2}{*}{0,034} \\
\hline Control & $-12,1(-17,8$ a $-6,4)$ & & & $-14,6(-20,4 \mathrm{a}-8,9)$ & & \\
\hline \multicolumn{7}{|l|}{$C T(\mathrm{mg} / \mathrm{dl})$} \\
\hline Intervención & $-22,1(-28,0$ a $-16,1)$ & $9,1(0,3$ a 17,9$)$ & \multirow{2}{*}{$0,042^{*}$} & $-25,4(-32,4 \mathrm{a}-18,4)$ & $9,7(0,4$ a 19,1$)$ & \multirow{2}{*}{$0,041^{*}$} \\
\hline Control & $-12,9(-19,5$ a $-6,4)$ & & & $-16,3(-22,5 \mathrm{a}-10,0)$ & & \\
\hline \multicolumn{7}{|l|}{$c H D L(m g / d l)$} \\
\hline Intervención & $0,8(-0,9$ a 2,6$)$ & $0,7(-2,2$ a 3,4$)$ & \multirow{2}{*}{ NS } & $0,1(-1,6$ a 1,9$)$ & $0,1(-2,6$ a 2,7$)$ & \multirow{2}{*}{ NS } \\
\hline Control & $0,2(-2,0$ a 2,4$)$ & & & $-0,1(-2,0$ a 1,9$)$ & & \\
\hline \multicolumn{7}{|c|}{ Triglicéridos $(\mathrm{mg} / \mathrm{dl})$} \\
\hline Intervención & $-3,5(-13,7$ a 6,6$)$ & $-7,9(-23,1$ a 8,2$)$ & \multirow{2}{*}{ NS } & $-4,2(-15,1$ a 6,8$)$ & $-3,4(-19,1$ a 12,4$)$ & \multirow{2}{*}{ NS } \\
\hline Control & $-11,7(-23,8$ a 0,4$)$ & & & $-7,5(-19,0$ a 3,9$)$ & & \\
\hline \multicolumn{7}{|l|}{ Peso (kg) } \\
\hline Intervención & $-0,41(-1,09$ a 0,29$)$ & $0,51(-0,62$ a 1,64$)$ & \multirow{2}{*}{ NS } & $-0,54(-1,26$ a 0,18$)$ & $0,56(-1,05$ a 2,16$)$ & \multirow{2}{*}{ NS } \\
\hline Control & $0,12(-0,80$ a 1,01$)$ & & & $0,02(-1,45$ a 1,49$)$ & & \\
\hline \multicolumn{7}{|l|}{ PAS ( $m m H g)$} \\
\hline Intervención & $-1,90(-4,18$ a 0,38$)$ & $0,84(-2,50$ a 4,19$)$ & \multirow{2}{*}{ NS } & $-2,97(-5,39$ a $-0,56)$ & $0,83(-2,67$ a 4,33$)$ & \multirow{2}{*}{ NS } \\
\hline Control & $-1,05(-3,52$ a 1,42$)$ & & & $-2,14(-4,69$ a 0,41$)$ & & \\
\hline \multicolumn{7}{|l|}{$P A D(m m H g)$} \\
\hline Intervención & $-0,88(-2,37$ a 0,60$)$ & $0,23(-1,79$ a 2,25$)$ & \multirow{2}{*}{ NS } & $-1,68(-3,24$ a $-0,13)$ & $1,64(-0,55$ a 3,84$)$ & \multirow{2}{*}{ NS } \\
\hline Control & $-0,65(-2,04$ a 0,73$)$ & & & $-0,04(-1,60$ a 1,52$)$ & & \\
\hline
\end{tabular}

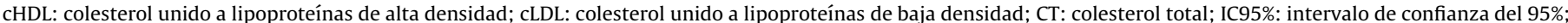
NS: no significativo; PAD: presión arterial diastólica; PAS: presión arterial sistólica.

Diferencia estadísticamente significativa en la reducción media entre los grupos de intervención y de control $(\mathrm{p}<0,05)$.

influir el alto porcentaje de participantes incluidos con un $\mathrm{RCV}<5 \%$. También podría contribuir la ausencia de efecto de la intervención sobre la adherencia a las recomendaciones dietéticas y su posible repercusión en el control lipídico.

Los resultados obtenidos coinciden tanto con los de estudios previos -que muestran una mejora del cumplimiento, pero no del control del $\mathrm{cLDL}^{27}$ - como con aquellos que demuestran reducción del cLDL sin mejorar su control ${ }^{28}$. Otras intervenciones con menos

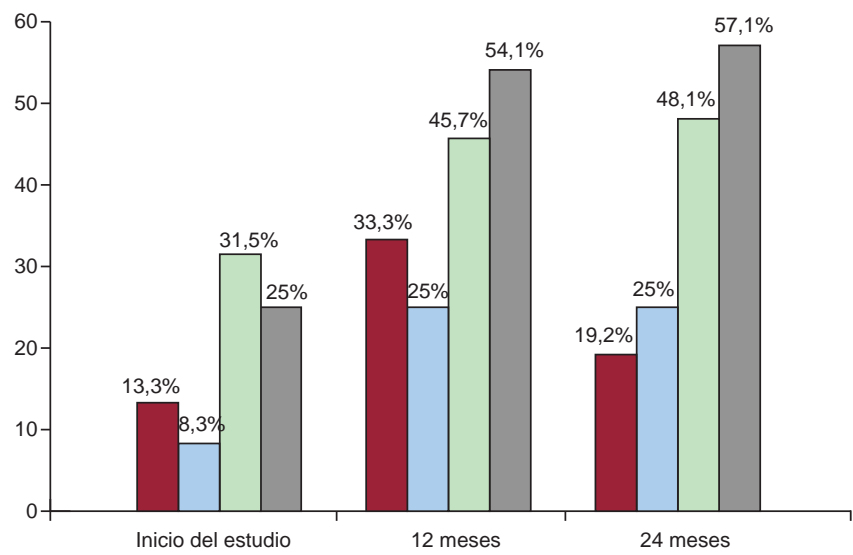

RCV muy alto $\square R C V$ alto $\square$ RVC moderado $\square R C V$ bajo

Figura 2. Porcentaje de pacientes del grupo que ha recibido la intervención que alcanza el objetivo terapéutico en cada subgrupo de RCV (SCORE) al inicio del estudio, a los 12 meses y a los 24 meses de seguimiento. RCV: riesgo cardiovascular. participantes e inferior seguimiento muestran mejora del cumplimiento y del control, de forma similar a los resultados obtenidos en el presente estudio al año de seguimiento ${ }^{13-15}$. Asimismo, hay estudios que han demostrado mejorar el control del perfil lipídico sin aumentar la adherencia ${ }^{29}$. Por otra parte, respecto al cumplimiento también se muestran resultados concordantes con ensayos previos al mejorar únicamente la adherencia a las recomendaciones sobre ejercicio, pero no sobre la dieta ${ }^{29}$.

Las diferencias con estudios previos incluyen -además del tipo de intervención- el tamaño muestral, la duración y las cifras de

Tabla 3

Evolución del cumplimiento terapéutico de los sujetos que continúan en el estudio en cada una de las visitas determinado mediante la prueba MoriskyGreen

\begin{tabular}{|c|c|c|c|c|}
\hline \multirow[t]{2}{*}{ Cumplimiento } & \multicolumn{2}{|c|}{12 meses } & \multicolumn{2}{|c|}{24 meses } \\
\hline & $\%$ & $\mathrm{p}$ & $\%$ & $\mathrm{p}$ \\
\hline Tratamiento hipolipemiante & $(n=223)$ & & $(n=220)$ & \\
\hline Grupo de intervención & 78,5 & \multirow{2}{*}{0,025} & 77,2 & \multirow{2}{*}{0,029} \\
\hline Grupo control & 64,9 & & 64,1 & \\
\hline Recomendaciones de ejercici & $(\mathrm{n}=314)$ & & $(n=304)$ & \\
\hline Grupo de intervención & 60,5 & \multirow{2}{*}{$<0,001$} & 64,9 & \multirow{2}{*}{$<0,001$} \\
\hline Grupo control & 38,3 & & 35,8 & \\
\hline Recomendaciones de dieta & $(n=314)$ & & $(n=304)$ & \\
\hline Grupo de intervención & 54,4 & \multirow{2}{*}{0,341} & 64,7 & \multirow{2}{*}{0,240} \\
\hline Grupo control & 49,0 & & 58,1 & \\
\hline
\end{tabular}

Porcentaje de cumplimiento.

Cómo citar este artículo: Párraga-Martínez I, et al. Eficacia de una estrategia combinada para mejorar el control del colesterol unido a lipoproteínas de baja densidad en pacientes con hipercolesterolemia. Ensayo clínico aleatorizado. Rev Esp Cardiol. 2017. http://dx.doi.org/10.1016/j.recesp.2017.03.019 
Tabla 4

Distribución de los pacientes según el fármaco hipolipemiante tomado al inicio del estudio y cambios de tratamiento realizados durante el seguimiento

\begin{tabular}{|c|c|c|c|c|}
\hline \multicolumn{5}{|c|}{ Consumo de fármacos hipolipemiantes al inicio } \\
\hline & Total $(n=243)$ & Intervención $(\mathrm{n}=114)$ & Control $(n=129)$ & $\mathrm{p}$ \\
\hline Simvastatina & $153(63,0)$ & $74(64,9)$ & $79(61,2)$ & 0,554 \\
\hline Atorvastatina & $67(27,6)$ & $32(28,1)$ & $35(27,1)$ & 0,870 \\
\hline \multirow[t]{3}{*}{ Otros } & $23(9,5)$ & $8(7,0)$ & $15(11,6)$ & 0,220 \\
\hline & \multicolumn{4}{|c|}{ Cambios de tratamiento hipolipemiante durante el seguimiento } \\
\hline & Total $(n=243)$ & Intervención $(\mathrm{n}=114)$ & Control $(n=129)$ & $\mathrm{p}$ \\
\hline Cambio de dosis o tipo de hipolipemiante & $31(12,7)$ & $15(13,3)$ & $16(12,2)$ & 0,804 \\
\hline Cambio de dosis & $17(6,9)$ & $7(6,1)$ & $10(7,6)$ & 0,633 \\
\hline \multirow[t]{2}{*}{ Cambio de fármaco } & $14(5,7)$ & $8(7,0)$ & $6(4,6)$ & 0,412 \\
\hline & Total $(n=358)$ & Intervención ( $\mathrm{n}=179)$ & Control $(n=179)$ & $\mathrm{p}$ \\
\hline $\begin{array}{l}\text { Cambio de dosis, tipo de fármaco o adición } \\
\text { de nuevo fármaco }\end{array}$ & $38(10,6)$ & $17(9,5)$ & $21(11,7)$ & 0,493 \\
\hline Adición de nuevo fármaco & $7(2,0)$ & $2(1,1)$ & $5(2,8)$ & 0,252 \\
\hline
\end{tabular}

Salvo otra indicación, los datos expresan $\mathrm{n}(\%)$.

cLDL consideradas como adecuado control. Se aportan los resultados a largo plazo de un ensayo multicéntrico sobre la eficacia de una intervención compuesta por 3 estrategias diferentes para reducir el cLDL, mejorar el cumplimiento y el grado de control según las guías europeas actuales. Los estudios previos utilizan otras intervenciones, en muchos casos únicas ${ }^{14}$, con menor tamaño muestral y frecuentemente con seguimiento inferior a 6 meses $^{13,15}$. También se ha comprobado ausencia de efectos adversos y elevada satisfacción con la intervención que no se han considerado en estudios previos ${ }^{13-15,29}$.

Respecto a la relevancia clínica de los resultados obtenidos, la reducción media de cLDL y $\mathrm{CT}$ no es muy relevante, aunque si superior a la de ensayos previos que evaluaron otras intervenciones $^{29}$, pudiendo disminuir el riesgo de morbimortalidad cardiovascular $^{3}$. Por tanto, el beneficio se debe tanto al control del cLDL como a la reducción de los valores séricos ${ }^{30}$. De hecho, el cLDL es la única variable que no ha mostrado tener un umbral inferior a partir del cual deje de observarse beneficio ${ }^{31}$, ocasionando que algunas guías no propongan un objetivo concreto del $\mathrm{CLDL}^{4}$. Por otra parte, aunque el porcentaje de pacientes con adecuado control del cLDL ha sido bajo, las guías recomiendan buscar las alternativas terapéuticas más económicas e implementar programas de prevención, implicando al paciente y facilitándole instrucciones escritas y verbales ${ }^{3,20}$. En consecuencia, esta intervención -que incluye actividades para mejorar el conocimiento sobre dislipemia, recordar indicaciones y facilitar mayor participación del paciente- podría adaptarse a esas recomendaciones y contribuir al mejor control del colesterol. Por último, aunque la reducción no ha sido muy relevante, estos resultados permiten considerar esta intervención en pacientes con hipercolesterolemia, facilitando el uso de dosis inferiores de estatinas y minimizando sus posibles efectos secundarios.

Tabla 5

Modelo de regresión logística para variables relacionadas con adecuado control del valor plasmático de colesterol unido a lipoproteínas de baja densidad a los 12 meses

\begin{tabular}{llr}
\hline Variables & OR (IC95\%) & \multicolumn{1}{l}{$\mathrm{p}$} \\
\hline Intervención combinada & $2,69(1,47-4,93)$ & 0,001 \\
\hline Menor edad (ser más joven) & $1,05(1,01-1,19)$ & 0,020 \\
\hline No presentar diabetes & $7,12(2,88-17,61)$ & $<0,001$ \\
\hline Hipertensión arterial & $1,94(1,02-3,69)$ & 0,044 \\
\hline Valor más bajo basal de cLDL en plasma $(\mathrm{mg} / \mathrm{dl})$ & $1,01(1,01-1,02)$ & 0,006 \\
\hline
\end{tabular}

cLDL: colesterol unido a lipoproteínas de baja densidad; IC95\%: intervalo de confianza del 95\%: OR: odds ratio.

\section{Limitaciones}

Entre las limitaciones cabe señalar que la intervención propuesta no permite aplicar las técnicas de enmascaramiento, aunque sí permite la evaluación ciega de los resultados. También sería posible que durante el seguimiento hubiera un riesgo de pérdidas diferenciales entre los grupos, aunque no hubo diferencias significativas. Las modificaciones de estatinas durante el seguimiento podrían representar una limitación, aunque no hubo diferencias entre los grupos; por lo que es poco probable que la mejora del control o las reducciones del perfil lipídico se debieran a cambios del tipo o de la dosis de estatinas. Asimismo, también podría ser una limitación el método para determinar el cumplimiento y, sin embargo, no hay un sistema gold standard que sea sencillo y del todo fiable ${ }^{32}$. Además, la guía NICE (2009) ${ }^{33}$ afirma que el cumplimiento autoinformado es una herramienta útil para la práctica clínica. Por otra parte, la medida utilizada como objetivo es la cifra de cLDL que es un método claramente objetivo. Por último, como en la mayoría de los ensayos, es posible que tanto médicos como participantes cambien su comportamiento por participar en el estudio, pudiendo explicarse de esta forma las mejoras también observadas en el grupo control (efecto-Hawthorne).

Probablemente sea necesario llevar a cabo nuevos estudios controlados multicéntricos de mayor duración para comprobar la efectividad de esta estrategia en la disminución de eventos cardiovasculares. También se precisan estudios con mayor potencia y diseñados específicamente para comprobar su efectividad en diferentes grupos de RCV, tal como parecen indicar los resultados en sujetos con RCV bajo o moderado. Además, es necesario conocer nuevas estrategias que incrementen tanto la adherencia farmacológica como las recomendaciones sobre ejercicio y dieta; pues la enfermedad cardiovascular se mantiene como primera causa de muerte, posiblemente por el aumento de prevalencia de estilos de vida poco saludables ${ }^{34,35}$. Asimismo, es necesario realizar estudios de coste-efectividad de estas intervenciones en los que se comparen las diferentes estrategias para aumentar el control y la adherencia.

\section{CONCLUSIONES}

Los resultados demuestran la eficacia de esta intervención -combinada y a largo plazo- en la reducción de las concentraciones plasmáticas de cLDL y en la mejora del cumplimiento terapéutico en pacientes con hipercolesterolemia. Además, mues- 
tran una mejora del control del cLDL al año. La reducción del cLDL superó el $10 \%$ respecto a los valores iniciales tanto al año como a los 2 años, y esto podría resultar clínicamente relevante.

\section{AGRADECIMIENTOS} nales.

Agradecemos la participación a los pacientes y a los profesio-

\section{FINANCIACIÓN}

Este estudio ha recibido financiación del Instituto de Salud Carlos III y del Fondo Europeo de Desarrollo Regional, Subprograma de Proyectos de Investigación en Salud (PI12/01955). Resolución de fecha 20 de diciembre de 2012.

\section{CONFLICTO DE INTERESES}

Ninguno.

\section{¿QUÉ SE SABE DEL TEMA?}

- El control del cLDL en pacientes con hipercolesterolemia es inferior al deseado, aunque hay un amplio consenso acerca de su tratamiento.

- La importancia de la adherencia terapéutica en dicho control se refleja en el incremento progresivo del espacio que ocupa en las sucesivas actualizaciones de las guías sobre hipercolesterolemia, e incluso es uno de sus puntos más destacados.

- La mejora del perfil lipídico depende del tratamiento farmacológico y de los estilos de vida, y el incumplimiento afecta tanto a uno como a otros.

\section{¿QUÉ APORTA DE NUEVO?}

- Mediante un ensayo clínico multicéntrico se ha demostrado la eficacia de una intervención en la reducción a largo plazo de las concentraciones plasmáticas de cLDL. Esa reducción alcanza cifras de relevancia clínica.

- Los resultados demuestran la mejora a largo plazo tanto del cumplimiento farmacológico como de las recomendaciones sobre ejercicio físico.

- Estos resultados muestran un mejor grado de control de los pacientes con hipercolesterolemia mediante una intervención compuesta por 3 actuaciones de sencilla aplicación en pacientes con RCV.

\section{MATERIAL SUPLEMENTARIO}

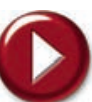

Se puede consultar material suplementario a este artículo en su versión electrónica disponible en http://dx.doi.org/ 10.1016/j.recesp.2017.03.019.

\section{BIBLIOGRAFÍA}

1. Grau M, Elosua R, Cabrera de León A, et al. Cardiovascular Risk Factors in Spain in the First Decade of the 21st Century, a Pooled Analysis With Individual Data From 11 Population-Based Studies: the DARIOS Study. Rev Esp Cardiol. 2011;64:295-304.
2. Guallar-Castillón P, Gil-Montero M, León-Muñoz LM, et al. Magnitud y manejo de la hipercolesterolemia en la población adulta de España, 2008-2010, estudio ENRICA. Rev Esp Cardiol. 2012;65:551-558.

3. Reiner Z, Catapano AL, De Backer C, et al. ESC/EAS Guidelines for the management of dyslipidaemias: the Task Force for the management of dyslipidaemias of the European Society of Cardiology (ESC) and the European Atherosclerosis Society (EAS). Eur Heart J. 2011;32:1769-1818.

4. Stone NJ, Robinson J, Lichtenstein AH, et al. 2013 ACC/AHA guideline on the treatment of blood cholesterol to reduce atherosclerotic cardiovascular risk in adults: a report of the American College of Cardiology/American Heart Association Task Force on Practice Guidelines. J Am Coll Cardiol. 2014;63:2889-2934.

5. Catapano AL, Graham I, De Backer C, et al. 2016 ESC/EAS Guidelines for the Management of Dyslipidaemias. Eur Heart J. 2016;37:2999-3058.

6. Reiner Z, De Bacquer D, Kotseva K, Prugger C, De Backer G, Wood D. Treatment potential for dyslipidaemia management in patients with coronary heart disease across Europe: findings from the EUROASPIRE-III survey. Atherosclerosis. 2013;231:300-307.

7. Kotseva K, Wood D, De Bacquer D, et al.; EUROASPIRE Investigators. EUROASPIREIV: A European Society of Cardiology survey on the lifestyle, risk factor and therapeutic management of coronary patients from 24 European countries. Eur J Prev Cardiol. 2016;23:636-648.

8. Anguita Sánchez M, Castro Conde A, Cordero Fort A, et al. Challenges in Oral Lipidlowering Therapy: Position Document of the Spanish Society of Cardiology. Rev Esp Cardiol. 2016;69:1083-1087.

9. Galve E, Cordero A, Cequier A, Ruiz E, González-Juanatey JR. Degree of Lipid Control in Patients With Coronary Heart Disease and Measures Adopted by Physicians. REPAR Study. Rev Esp Cardiol. 2016;69:931-938.

10. Banegas JR, López-García E, Dallongeville J, et al. Achievement of treatment goals for primary prevention of cardiovascular disease in clinical practice across Europe: the EURIKA study. Eur Heart J. 2011;32:2143-2152.

11. Sclar DA, Chin A, Skaer TL, Okamoto MP, Nakahiro RK, Gill MA. Effect of health education in promoting prescription refill compliance among patients with hypertension. Clin Ther. 1991;13:489-495.

12. Munoz MA, Vila J, Cabañero M, et al.; ICAR (Intervención en la Comunidad de Alto Riesgo cardiovascular) investigators. Efficacy of an intensive prevention program in coronary patients in primary care, a randomised clinical trial. Int J Cardiol. 2007; $118: 312-320$

13. Márquez Contreras E, Casado Martínez JJ, Corchado Albalat Y, et al. Eficacia de una intervención para mejorar el cumplimiento terapéutico en las dislipemias. Aten Primaria 2004:33:443-450.

14. Faulkner MA, Wadibia EC, Lucas BD, Hilleman DE. Impact of pharmacy counseling on compliance and effectiveness of combination lipid-lowering therapy in patients undergoing coronary artery revascularization: a randomized, controlled trial. Pharmacotherapy. 2000;20:410-416.

15. Márquez Contreras E, Casado Martínez JJ, Motero Carrasco J, et al. El cumplimiento terapéutico en las dislipemias medido mediante monitores electrónicos. ¿Es eficaz un calendario recordatorio para evitar los olvidos? Aten Primaria. 2007;39: 661-668

16. Palop Larrea V, Martínez Mir I. Adherencia al tratamiento en el paciente anciano. Inf Ter Sist Nac Salud. 2004;28:113-120.

17. McDonald HP, Garg AX, Haynes RB. Intervention to enhance patient adherente to medication prescriptions: scientific review. JAMA. 2002;288:2868-2879.

18. Maiques Galán A, Brotons Cuixart C, Villar Alvarez F, et al. Recomendaciones preventivas cardiovasculares PAPPS-2009. Programa de actividades preventivas y de promoción de la salud. Sociedad Española de Medicina de Familia y Comunitaria [consultado 5 Jun 2016]. Barcelona: semFYC; 2009. Disponible en: http:/ www.papps.es/upload/file/09\%20PAPPS\%20ACTUALIZACION\%202009.pdf.

19. Kotseva K, Wood D, De Backer G, De Bacquer D, Pyörälä K, Keil U. Cardiovascular prevention guidelines in daily practice: a comparison of EUROASPIRE-I, II, and III surveys in eight European countries. Lancet. 2009;373:929-940.

20. Perk J, De Backer G, Gohlke H, et al. European Guidelines on cardiovascular disease prevention in clinical practice (version 2012). The Fifth Joint Task Force of the European Society of Cardiology and Other Societies on Cardiovascular Disease Prevention in Clinical Practice (constituted by representatives of nine societies and by invited experts). Eur Heart J. 2012;33:1635-1701.

21. Domingo-Salvany A, Bacigalupe A, Carrasco JM, Espelt A, Ferrando J, Borrell C; Grupo de Determinantes Sociales de la Sociedad Española de Epidemiología. Propuestas de clase social neoweberiana y neomarxista a partir de la Clasificación Nacional de Ocupaciones 2011. Gac Sanit. 2013;27:236-272.

22. Morisky DE, Green LW, Levine DM. Concurrent and predictive validity of a selfreport measure of medication adherence. Med Care. 1986;24:67-74.

23. Marrugat J, Vila J, Baena-Diez JM, et al. Relative Validity of the 10-Year Cardiovascular Risk Estimate in a Population Cohort of the REGICOR Study. Rev Esp Cardiol. 2011:64:385-394.

24. Cordero A, Bertomeu-Martínez V, Mazón P, et al. Patients with cardiac disease: Changes observed through last decade in out-patient clinics. World J Cardiol. 2013;5:288-294.

25. Plana N, Ibarretxe D, Cabré A, Ruiz E, Masana L. Prevalence of atherogenic dyslipidemia in primary care patients at moderate-very high risk of cardiovascular disease. Cardiovascular risk perception. Clin Invest Arterioscler. 2014;26:274-284

26. Gielen S, Landmesser U. The Year in Cardiology 2013: cardiovascular disease prevention. Eur Heart J. 2014:35:307-312.

27. Jover JL, Gil VF, Tortajada JL, Mora C, Giner C, Merino J. Efecto de la entrevista motivacional sobre la cumplimentación de la terapéutica farmacológica en pacientes con dislipemia. Med Clin (Barc). 2001;116 Supl 2:137-140.

Cómo citar este artículo: Párraga-Martínez I, et al. Eficacia de una estrategia combinada para mejorar el control del colesterol unido a lipoproteínas de baja densidad en pacientes con hipercolesterolemia. Ensayo clínico aleatorizado. Rev Esp Cardiol. 2017. http://dx.doi.org/10.1016/j.recesp.2017.03.019 
28. Villeneuve J, Genest J, Blais L, et al. A cluster randomized controlled Trial to Evaluate an Ambulatory primary care Management program for patients with dyslipidemia: the TEAM study. CMAJ. 2010;182:447-455.

29. Bóveda-Fontán J, Barragán-Brun N, Campiñez-Navarro M, et al.; Collaborative Group Estudio Dislip-EM. Effectiveness of motivational interviewing in patients with dyslipidemia: randomized cluster trial. BMC Fam Pract. 2015;16:151.

30. Cordero A, López-Palop R, Bertomeu-González V, Carrillo P, Moreno-Arribas J, Bertomeu-Martínez V. Perfil clínico y pronóstico de los pacientes con síndrome coronario agudo y colesterol unido a lipoproteínas de baja densidad $<70 \mathrm{mg} / \mathrm{dl}$. Rev Esp Cardiol. 2013;66:588-589.

31. Baigent C, Blackwell L, Emberson J, et al. Efficacy and safety of more intensive lowering of LDL cholesterol: a meta-analysis of data from 170,000 participants in 26 randomised trials. Lancet. 2010;376:1670-1681.
32. Vermeire E, Hearnshaw H, Van Royen P, Denekens J. Patient adherence to treatment: three decades of research. A comprehensive review. I Clin Pharm Ther 2001;26:331-342.

33. National Collaborating Centre for Primary Care. Medicines adherence: involving patients in decisions about prescribed medicines and supporting adherence. Londres: NICE; 2009 [consultado 5 Jun 2016]. Disponible en: https://www.nice.org.uk/ guidance/cg76.

34. World Health Statistics 2012. Ginebra: World Health Organization; 2012 [consultado 15 Jun 2016]. Disponible en: http://apps.who.int/iris/bitstream/10665/ 44844/1/9789241564441_eng.pdf.

35. Dégano IR, Elosua R, Marrugat J. Epidemiología del síndrome coronario agudo en España: estimación del número de casos y la tendencia de 2005 a 2049. Rev Esp Cardiol. 2013;66:472-481. 